\title{
INFLUÊNCIA DO ÁCIDO INDOLBUTÍRICO NO ENRAIZAMENTO DE ESTACAS APICAIS E BASAIS DE CARAMBOLEIRA (Averrhoa carambola L.) SOB CONDIÇÕES DE NEBULIZAÇÃO INTERMITENTE ${ }^{1}$
}

\author{
DÉBORA COSTA BASTOS², ANTONIO BALDO GERALDO MARTINS ${ }^{3}$, ERIVALDO JOSÉ SCALOPPI JUNIOR ${ }^{4}$, \\ ISABELE SARZI ${ }^{5}$, JÚNIOR CÉSAR FATINANSI ${ }^{6}$
}

\begin{abstract}
RESUMO - A produção de mudas de caramboleira é um dos fatores limitantes à expansão comercial da cultura, devido ao tempo que estas levam para serem formadas e iniciarem a produção. Uma boa alternativa pode estar na otimização dos métodos de propagação vegetativa, através de estudos relativos aos processos e fatores envolvidos no enraizamento de estacas. Este trabalho foi desenvolvido com o objetivo de estudar o enraizamento de estacas apicais e basais de caramboleira, tratadas com ácido indolbutírico (IBA), em condições de nebulização intermitente. Estacas apicais e basais de caramboleira foram coletadas de ramos de plantas-matrizes da cultivar B-10 e submetidas à aplicação de cinco concentrações de IBA (0; 1.000; 3.000; 5.000 e $7000 \mathrm{mg} . \mathrm{L}^{-1}$ ), em imersão por 10 segundos, para avaliar a capacidade de sobrevivência, enraizamento e número médio de raízes/estaca. Posteriormente, as estacas foram colocadas em caixas de madeira contendo vermiculita média como substrato e mantidas em casa de vegetação, sob nebulização intermitente, durante 70 dias. O delineamento experimental utilizado foi em esquema fatorial $2 \times 5$, com 4 repetições e 10 estacas/parcela. As estacas apicais obtiveram melhores resultados para porcentagem de sobrevivência $(49,26 \%)$, enraizamento (34,84\%) e número médio de raízes (20,51), mostrando-se superiores às basais. O uso de IBA não influenciou em nenhuma das variáveis analisadas.
\end{abstract}

Termos para indexação: propagação vegetativa, regulador de crescimento.

\section{INFLUENCE OF INDOL BUTYRIC ACID ON THE ROOTING OF TOP AND BASE CUTTINGS OF STAR FRUIT (Averrhoa carambola L.) UNDER INTERMITENT MIST}

\begin{abstract}
Star fruit seedling production is one of the drawbacks to the commercial expansion due to the length of time it takes for their formation and production. An alternative would be the improvement of vegetative propagation methods studing the processes and factors concerned with rooting of cuttings. This paper was carried out with the objective to study rooting of top and base star fruit cuttings, treated with Indol Butyric Acid (IBA) concentrations under intermittent mist. Top and base cuttings of star fruit ' $\mathrm{B}-10$ ' were submitted to five concentrations of IBA( $(0,1000,3000$, 5000 , and $7000 \mathrm{mg} . \mathrm{L}^{-1}$ ), for 10 seconds, to verify survival and rooting capacity, and medium root number per cuttings. Later the cuttings were placed in wooden boxes containing vermiculite as substrate and maintained in greenhouse under intermittent mist for 70 days. The experimental design was completely randomized, with the factorial $2 \times 5$, with 4 replications and 10 cuttings each plot. Top cuttings obtained better results for survival percentage (49.26\%), rooting percentage (34.84\%), and medium root number per cuttings (20.51), than base cuttings. None of variables analyzed here were affected by the use of IBA.
\end{abstract}

Index terms: vegetative propagation, growth regulator.

\section{INTRODUÇÃO}

A caramboleira é uma planta originária da Ásia e típica das regiões tropicais de ambos os hemisférios. É uma planta exótica, cultivada por quase todo o Brasil, com exceção das zonas mais frias (Oliveira et al., 1989).

É considerada como fruteira com potencial de exploração, devido ao rápido desenvolvimento, à alta produtividade, à existência de variedades doces, à possibilidade de contornar fatores limitantes ao cultivo, à baixa exigência quanto aos tratos culturais (solo, adubação, tratamentos fitossanitários, controle de plantas daninhas e outros) e ao fato de produzir frutos com aparência e sabor únicos. Trabalhos de marketing, apresentação, degustação e forma de consumo dos frutos, aliados à sua forma atrativa, promoveram e conquistaram os consumidores do mundo (Sauco et al., 1993).

Os principais produtores mundiais de carambola, em ordem decrescente, são Taiwan (1/3 da produção mundial), Malásia e Brasil. Neste último, a produção anual é estimada em 3.000 T, proveniente, em sua maioria, de pomares domésticos (Donadio et al., 2001).

Na última década, em função da diversificação e do interesse crescente por frutas exóticas, surgiram plantios comerciais no Estado de São Paulo, os quais vêm, cada vez mais, se expandindo. A carambola pode ser consumida in natura, forma em que vem tendo destaque, ou usada na fabricação de compotas ou geléias, mas ainda em estágio artesanal (Donadio et al., 2001).

A produção de mudas da caramboleira pode ser por meio de sementes ou enxertia (borbulhia ou garfagem). A utilização de sementes no processo de multiplicação é inviável devido à ocorrência de variabilidade genética, pequena viabilidade das sementes e ao grande período para iniciar a produção, devendo ser utilizada apenas para a formação dos porta-enxertos (Nakasone \& Paul, 1998).

Embora a enxertia seja o método mais utilizado comercialmente, apresenta dificuldades, como o grande período que as mudas levam para estarem em condições de serem plantadas no local definitivo, problemas de incompatibilidade, maior mão-de-obra e alto custo (Donadio et al., 2001).

Em vista disso, têm sido estudados outros métodos de propagação que possam ser viáveis. Dentre eles, a estaquia em câmara de nebulização é o que tem sido amplamente utilizado em fruticultura, porém o sucesso de sua utilização é variável por uma série de fatores de natureza genética e ambiental.

O processo de formação de raízes em estacas é influenciado por um grande número de fatores que podem atuar isoladamente ou em conjunto. Dentre esses, destacam-se as condições fisiológicas da planta-

\footnotetext{
${ }^{1}$ (trabalho 153/2003). Recebido: 17/10/2003. Aceito para publicação: 14/05/2004. Parte da Dissertação do primeiro autor. Apoio financeiro FAPESP. Trabalho (153/ 03).

${ }^{2}$ Eng $^{\mathrm{a}}$. Agr ${ }^{\mathrm{a}}$., MSc., Doutoranda em Fitotecnia, Departamento de Produção Vegetal, USP/ESALQ, Caixa Postal 9, CEP 13418-900, Piracicaba-SP. dcbastos@ @esalq.usp.br. ${ }^{3}$ Eng. Agr. Professor Doutor do Departamento de Produção Vegetal, FCAV/UNESP, Via de Acesso Prof. Paulo Donato Castelanni, s/n, CEP 14884-900, JaboticabalSP.baldo@fcav.unesp.br

${ }^{4}$ Eng. Agr. Doutorando do Departamento de Produção Vegetal, FCAV/UNESP, Jaboticabal-SP.

${ }^{5}$ Eng $^{\mathrm{a}}$. Agr ${ }^{\mathrm{a}}$. Doutoranda do Departamento de Horticultura, FCA/UNESP, Botucatu-SP.

${ }^{6}$ Engenheiro Agrícola, Mestrando em Ecologia de Agroecossistemas, ESALQ/USP, Caixa Postal 9, CEP 13418-900, Piracicaba-SP.
} 
matriz (presença de carboidratos, substâncias nitrogenadas, aminoácidos, auxinas, compostos fenólicos e outras substâncias não identificadas), o período e posição de coleta das estacas, juvenilidade, estiolamento, presença de folhas e gemas, idade da planta-matriz e fatores do ambiente, como disponibilidade de água, luminosidade e substrato (Hartmann et al., 1990).

A caramboleira apresenta resultados bastante variáveis em relação ao processo de enraizamento de estacas. Bastos (2002), estudando a estaquia de caramboleira, sob condições de nebulização intermitente, obteve $63 \%$ de enraizamento utilizando estacas herbáceas apicais tratadas com $5.000 \mathrm{mg} . \mathrm{L}^{-1}$ de IBA no período de verão; porém, estes resultados não se repetiram quando as estacas utilizadas eram lenhosas. Este mesmo autor, testando quatro concentrações do regulador de crescimento em imersão lenta, observou maior potencial de enraizamento $(37,5 \%)$ nas estacas apicais coletadas na época do verão, sem o tratamento com ácido indolbutírico. Mediante esses resultados, existe a necessidade de desenvolver outras pesquisas nesta área.

O objetivo deste trabalho foi estudar o efeito do ácido indolbutírico no enraizamento de estacas apicais e basais de caramboleira, sob condições de nebulização intermitente.

\section{MATERIAL E MÉTODOS}

O presente trabalho foi conduzido na área de produção de mudas do Sítio São João, em dezembro de 2000, situado no município de Taquaritinga-SP, localizado a $21^{\circ} 44^{\prime} 44^{\prime \prime}$ de latitude sul e a $48^{\circ} 29^{\prime} 53^{\prime \prime}$ de longitude a oeste, com altitude de $512 \mathrm{~m}$. O clima da região pode ser classificado, segundo Köeppen, como Cwa, subtropical com chuvas de verão, e inverno relativamente seco. A média das temperaturas durante a realização do experimento foi de $24,5^{\circ} \mathrm{C}$ e da umidade relativa do ar de $76,5 \%$.

Estacas apicais e basais de caramboleira cultivar B-10 foram coletadas de plantas-matrizes com 15 anos de idade, na região mediana da copa, em pleno desenvolvimento vegetativo, e preparadas com $12 \mathrm{~cm}$ de comprimento e 1 par de folhas inteiras. Os ramos utilizados para a coleta das estacas foram herbáceos, de "madeira verde".

As estacas foram submetidas à aplicação de cinco concentrações de IBA $(0 ; 1.000 ; 3.000 ; 5.000$ e 7.000 mg.L.-1), através da imersão da base por 10 segundos, sendo estaqueadas em caixas de madeira tipo uva $(24,0$ x 45,0 x 10,0 cm) contendo vermiculita de grânulos médios, em casa de vegetação, sob nebulização intermitente, com proteção apenas nas laterais e a pleno sol (ambiente sem nemhum tipo de cobertura, ficando as estacas expostas ao sol, chuva, vento, etc.). O tempo de nebulização foi determinado com a finalidade de manter uma fina camada de água sobre a superfície das folhas, sendo de 15 segundos espaçados de 45 segundos, controlados por um temporizador, o qual regulava a abertura e o fechamento da válvula solenóide, por 24 horas seguidas e durante todo o tempo de condução do experimento.

$\mathrm{O}$ delineamento experimental utilizado foi o inteiramente casualizado, num esquema fatorial 2 x 5 ( 2 tipos de estacas quanto ao estádio vegetativo e 5 concentrações de IBA), com 4 repetições de 10 estacas cada. Os resultados foram submetidos à análise de variância pelo teste de F. Os dados de porcentagem foram transformados em arcsen $\sqrt{x+0,5}$

Após 70 dias de instalação do experimento, avaliaram-se a porcentagem de sobrevivência, enraizamento e número médio de raízes.

\section{RESULTADOS E DISCUSSÃO}

Na Tabela 1, verificam-se os valores médios dos tipos de estacas referentes às concentrações de IBA aplicadas. Quanto à capacidade de enraizamento, observa-se que as estacas apicais $(34,84 \%)$ apresentaram melhores resultados em relação às estacas basais $(4,79 \%)$. Tal fato pode ser atribuído ao maior grau de lignificação das estacas basais que parece estar correlacionado negativamente com o nível de auxina, estando as enzimas como as peroxidases envolvidas na síntese de lignina e degradação de auxinas (Ono \& Rodrigues, 1996). Segundo Hartmann et al. (1990), tecidos mais lignificados também podem funcionar como barreira física para a emissão de raízes. Resultados semelhantes foram observados por Bastos (2002), que verificou que estacas menos lignificadas de caramboleira (herbáceas) apresentaram melhores resultados para enraizamento, numero médio de raízes e sobrevivência, em relação às estacas mais lignificadas (lenhosas), confirmando o encontrado nesse trabalho.

TABELA 1- Porcentagens de sobrevivência e de enraizamento e número médio de raízes de estacas apicais e basais de caramboleira (Averrhoa carambola L.), FCAV/UNESP, Jaboticabal-SP, 2002.

\begin{tabular}{cccc}
\hline Tipos de estaca & $\begin{array}{c}\text { Sobrevivência } \\
(\%)\end{array}$ & $\begin{array}{c}\text { Enraizamento } \\
(\%)\end{array}$ & $\begin{array}{c}\text { Número Médio } \\
\text { de Raízes }\end{array}$ \\
\hline Estacas apicais & $49,26 \mathrm{a}^{*}$ & $34,84 \mathrm{a}$ & $20,51 \mathrm{a}$ \\
Estacas basais & $18,72 \mathrm{~b}$ & $4,79 \mathrm{~b}$ & $1,6 \mathrm{~b}$ \\
\hline CV $(\%)$ & 25,72 & 39,79 & 76,22 \\
\hline
\end{tabular}

* Médias seguidas por letras distintas diferem entre si, ao nível de 5\%, pelo teste $\mathrm{F}$ da análise de variância.

Houve superioridade das estacas apicais $(49,26 \%)$ em relação às basais $(18,72 \%)$ quanto à porcentagem de sobrevivência (Tabela 1). Isso provavelmente ocorreu pelo fato de as estacas apicais apresentarem maior capacidade de enraizamento; a presença de raízes permite que as estacas absorvam nutrientes necessários a sua manutenção, evitando, portanto, um esgotamento das reservas e a sua morte. Porém, Hartmann et al. (1990) e Nachtigal (1999) concluíram que as estacas menos lignificadas (herbáceas) são mais sensíveis à desidratação e à morte. $\mathrm{O}$ baixo índice de enraizamento, a permanência prolongada na câmara de nebulização e o excesso de umidade são fatores que influenciam na sobrevivência das estacas.

Quanto ao número médio de raízes (Tabela 1), as estacas apicais $(20,51)$ apresentaram resultados significativamente superiores aos das basais $(1,6)$, confirmando o resultado encontrado por Nachtigal (1999) em pessegueiro e por Bastos (2002) em caramboleira.

A utilização de IBA não teve efeito significativo na porcentagem de enraizamento (Tabela 2), concordando com os resultados encontrados por Leonel \& Rodrigues (1993) e Biasi et al. (1997) em videira, que não observaram aumento significativo no enraizamento de estacas com a aplicação desse regulador, sob condições controladas. Prati et al. (1999), em lima-ácida 'Tahiti', e Bastos (2002), em caramboleira, observaram não ser necessário o emprego de reguladores de crescimento para o enraizamento de estacas.

Em relação às concentrações de IBA (Tabela 2), pode-se observar que não houve efeito significativo na sobrevivência das estacas. Resultados semelhantes foram encontrados por Martins (1998), em lichieira, que verificou não haver influência positiva do IBA na sobrevivência das estacas. Roncatto et al. (1999) e Roberto et al. (2001), estudando o efeito do IBA no enraizamento de estacas de laranjeira 'Valência', verificaram que não houve influência desse regulador de crescimento na porcentagem de estacas sobreviventes.

TABELA 2 - Porcentagens de sobrevivência e de enraizamento e número médio de raízes de estacas de caramboleira (Averrhoa carambola L.), tratadas com diferentes concentrações de IBA, FCAV/UNESP, Jaboticabal-SP, 2002

\begin{tabular}{cccc}
\hline IBA $\left(\mathrm{mg.L}^{-1}\right)$ & $\begin{array}{c}\text { Sobrevivência } \\
(\%)\end{array}$ & $\begin{array}{c}\text { Enraizamento } \\
(\mathbf{\%})\end{array}$ & $\begin{array}{c}\text { Número Médio } \\
\text { de Raízes }\end{array}$ \\
\hline $\mathbf{0}$ & $38,56 *$ & 18,92 & 12,75 \\
$\mathbf{1 . 0 0 0}$ & 37,44 & 23,57 & 8,73 \\
$\mathbf{3 . 0 0 0}$ & 28,66 & 17,88 & 15,28 \\
$\mathbf{5 . 0 0 0}$ & 35,16 & 21,66 & 7,23 \\
$\mathbf{7 . 0 0 0}$ & 30,14 & 17,06 & 11,28 \\
\hline CV $(\%)$ & 25,72 & 39,79 & 76,22 \\
\hline
\end{tabular}

* Médias não diferem pelo teste $\mathrm{F}$ da análise de variância. 
A aplicação de IBA(Tabela 2) não apresentou efeito significativo em nenhuma das variáveis analisadas, concordando com Rossal (1994) que, avaliando o efeito do ácido indolbutírico sobre enraizamento de dois tipos de estaca de laranjeira 'Valência', mantidas em nebulização intermitente, verificou que a formação de raízes não foi estimulada pelos tratamentos realizados. Bastos (2002) também não observou efeito significativo da aplicação de IBA na formação de raízes em estacas herbáceas e lenhosas de caramboleira.

\section{CONCLUSÃO}

Nas condições em que o experimento foi realizado, pode-se concluir que:

Para a propagação vegetativa da caramboleira, podem ser utilizadas estacas herbáceas apicais com 1 par de folhas, não sendo necessária a utilização de IBA.

\section{REFERÊNCIAS BIBLIOGRÁFICAS}

BASTOS, D.C. Efeito da época de coleta, estádio do ramo e do tratamento com IBA no enraizamento de estacas de caramboleira (Averrhoa carambola L.). 2002. 75f. Dissertação (Mestrado em Agronomia) Faculdade de Ciências Agrárias e Veterinárias, Universidade Estadual Paulista, Jaboticabal, 2002.

BIASI, L.A.; POMMER, C.V.; PINO, P.A.G.S. Propagação de portaenxertos de videira mediante estaquia semilenhosa. Bragantia, Campinas, v.56, n.2, p. 367-376, 1997.

DONADIO, L.C.; SILVA, J.A.A.; ARAÚJO, P.R.S.; PRADO, R.M. Caramboleira (Averrhoa carambola L.). Jaboticabal: Sociedade Brasileira de Fruticultura, 2001. 81p. (Série Frutas Potenciais).

HARTMANN, H.T.; KESTER, D. E.; DAVIES, JR, F.T. Plant propagation: principles and pratices. 5. ed. New Jersey: Prentice Hall, 1990. 647p.

LEONEL, S.; RODRIGUES, J.D. Efeito da época de estaquia, fitorreguladores e ácido bórico no enraizamento de estacas de portaenxertos de videira. Scientia Agricola, Piracicaba, v.50, n.1, p.27-32, 1993.
MARTINS, A. B. G. Enraizamento de estacas enfolhadas de três variedades de lichia (Litchi chinensis Sonn.). 1998. 100f. Tese (Doutorado em Agronomia) - Faculdade de Ciências Agrárias e Veterinárias, Universidade Estadual Paulista, Jaboticabal, 1998.

NACHTIGAL, J.C. Obtenção de porta-enxertos 'Okinawa' e de mudas de pessegueiro (Prunus persica $\left(L_{\text {. }}\right.$ ) Batsch) utilizando métodos de propagação vegetativa. 1999. 165f. Tese (Doutorado em Agronomia) - Faculdade de Ciências Agrárias e Veterinárias, Universidade Estadual Paulista, Jaboticabal, 1999.

NAKASONE, H.Y.; PAUL, R.E. Tropical fruits crop production science in horticulture. New York: Cab International, 1998. 445p.

OLIVEIRA, M. N.; MAIA, G. A.; GUEDES, Z. B. de L.; GUIMARÃES, A. C. L.; FIGUEIREDO, R. W. de. Características químicas e físicoquímicas da carambola (Averrhoa carambola L.), Ciência Agronômica, Fortaleza, v.20, n.1/2, p. 129- 133, 1989.

ONO, E.O.; RODRIGUES, J.D. Aspectos da fisiologia do enraizamento de estacas caulinares. Jaboticabal: FUNEP, 1996. 83p.

PRATI, P.; MOURÃO FILHO, F.A.A.; DIAS, C.T.S.; SCARPARE FILHO, J.A. Estaquia semilenhosa: um método rápido e alternativo para a produção de mudas de lima-ácida 'Tahiti'. Scientia Agricola, Piracicaba, v.56, n.1, p.185-190, 1999.

ROBERTO, S.R.; PEREIRA, F.M.; CAETANO, A.C. Efeito do ácido indolbutírico no enraizamento de estacas herbáceas de laranjeira 'Valência' (Citrus sinensis L. Osbeck). Revista Brasileira de Fruticultura, Jaboticabal, v.23, n.1, p. 206-208. Abril, 2001.

RONCATTO, G.; GONÇALVES, E.D.; DUTRA, L.F.; KERSTEN, E. Influência do sombreamento das plantas e do ácido indolbutírico no enraizamento de estacas de laranjeira (Citrus sinensis L. Osbeck) Cv. Valência. Revista Científica Rural, Pelotas, v.4, n.2, p.60-65, 1999.

ROSSAL, P.A.L. Efeito do ácido indolbutírico no enraizamento de estacas de laranjeira (Citrus sinensis L. Osbeck) cv. Valência sob condições de nebulização. 1994. 55f. Dissertação (Mestrado em Agronomia) Faculdade de Agronomia Eliseu Maciel, Universidade Federal de Pelotas, Pelotas, 1994.

SAUCO, V.S.; MENINI, U.G.; TINDALL, H.D. Carambola cultivation. Rome: FAO, 1993.74p. 